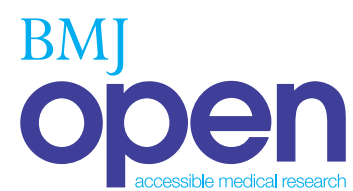

\title{
The effect of weekly short message service communication on patient retention in care in the first year after HIV diagnosis: study protocol for a randomised controlled trial (WelTel Retain)
}

Mia L van der Kop, ${ }^{1,2}$ David I Ojakaa, ${ }^{3}$ Anik Patel, ${ }^{4}$ Lehana Thabane, ${ }^{5}$ Koki Kinagwi, ${ }^{3}$ Anna Mia Ekström, ${ }^{2}$ Kirsten Smillie, ${ }^{6}$ Sarah Karanja, ${ }^{7}$ Patricia Awiti, ${ }^{2}$ Edward Mills, ${ }^{8}$ Carlo Marra, ${ }^{4}$ Lennie Bazira Kyomuhangi, ${ }^{3}$ Richard T Lester ${ }^{1}$

To cite: van der Kop ML, Ojakaa DI, Patel A, et al. The effect of weekly short message service communication on patient retention in care in the first year after HIV diagnosis: study protocol

for a randomised controlled trial (WelTel Retain). BMJ Open 2013;3:e003155. doi:10.1136/bmjopen-2013003155

- Prepublication history for this paper is available online. To view these files please visit the journal online (http://dx.doi.org/10.1136/ bmjopen-2013-003155).

Received 3 May 2013 Accepted 7 May 2013

This final article is available for use under the terms of the Creative Commons Attribution Non-Commercial 2.0 Licence; see http://bmjopen.bmj.com

For numbered affiliations see end of article.

Correspondence to Mia L van der Kop; miavanderkop@gmail.com

\section{ABSTRACT}

Introduction: Interventions to improve retention in care after HIV diagnosis are necessary to optimise the timely initiation of antiretroviral therapy (ART) and HIV/AIDS control outcomes. Widespread mobile phone use presents new opportunities to engage patients in care. A randomised controlled trial (RCT), WelTel Kenya1, demonstrated that weekly text messages led to improved ART adherence and viral load suppression among those initiating ART. The aim of this study was to determine whether the WelTel intervention is an effective and cost-effective method of improving retention in care in the first year of care following HIV diagnosis.

Methods and analysis: WelTel Retain is an open, parallel group RCT that will be conducted at the Kibera Community Health Centre in Nairobi, Kenya. Over a 1 -year period, we aim to recruit 686 individuals newly diagnosed with HIV who will be randomly allocated to an intervention or control arm (standard care) at a 1:1 ratio. Intervention arm participants will receive the weekly WeITel SMS 'check-in' to which they will be instructed to respond within $48 \mathrm{~h}$. An HIV clinician will follow-up and triage any problems that are identified. Participants will be followed for 1 year, with a primary endpoint of retention in care at 12 months. Secondary outcomes include retention in stage $1 \mathrm{HIV}$ care (patients return to the clinic to receive their first CD4 results) and timely ART initiation. Cost-effectiveness will be analysed through decision-analytic modelling.

Ethics and dissemination: Ethical approval has been obtained from the University of British Columbia and the African Medical and Research Foundation. This trial will test the effectiveness and cost-effectiveness of the WelTel intervention to engage patients during the first year of HIV care. Trial results and economic evaluation will help inform policy and practice on the use of WelTel in the early stages of HIV care.

Trial registration: ClinicalTrials.gov NCT01630304.

\section{ARTICLE SUMMARY}

Article focus

- Improving retention in care in the first year after HIV diagnosis is critical to optimise patienthealth outcomes; few interventions specifically designed to retain patients in the early stages of HIV care have been tested.

- This article outlines a randomised controlled trial protocol to determine the effectiveness and costeffectiveness of a text-messaging intervention (WelTel) to retain patients in HIV care in the first year after diagnosis.

Key messages

- The WelTel intervention has been found to be effective at promoting adherence among HIV patients taking antiretroviral therapy; this trial will determine whether the intervention is also effective in earlier stages along the continuum of HIV care.

- Mobile health interventions need to be evidencebased and rigorously tested before they are considered for implementation and scale-up.

Strengths and limitations of this study

- The trial has a tracing component to ascertain the true outcome of participants initially deemed to be 'lost-to-follow-up', leading to a more accurate assessment of retention in care.

- The primary limitation is that WelTel Retain is a single-site study in a resource-limited setting; generalisability to other settings may be limited.

\section{INTRODUCTION}

As HIV care and treatment programmes in resource-limited settings move away from emergency delivery of care to long-term management, patient retention in care is increasingly being recognised as critical to the success 
of these programmes. Along the cascade of care (figure 1), from diagnosis to clinical evaluation and readiness for antiretroviral therapy (ART), through to long-term follow-up, high rates of attrition from care occur at several intervals. Between diagnosis and initiation of ART, fewer than two-thirds of patients remain continuously in care. ${ }^{1}$ In Kenya, a study at a large HIV-care centre estimated 12-month clinic retention among ART-ineligible patients at $63 \%$; after introducing the provision of free co-trimoxazole to pre-ART patients, 12-month retention increased to over $80 \% .^{2}$ Consequences of attrition include an increased risk of morbidity, mortality and a greater strain on limited healthcare resources (resulting from patients returning to care when they are seriously ill). ${ }^{3} 4$ Conversely, retaining patients in care before they start HIV treatment facilitates the timely initiation of ART, as well as the provision of opportunistic infection prophylactic medication and prevention of mother-to-child transmission (PMTCT) services. A review of individual studies indicates that few interventions specifically designed to promote retention in care among individuals with HIV have been tested. Of these, financing transportation costs (N Emenyonu et al 17th Conference on Retroviruses and Opportunistic Infections, San Francisco, 2010), targeted social support (V Otieno et al, 17th International AIDS Conference, Mexico City, 2008) and the provision of free co-trimoxazole as prophylaxis for opportunistic infections ${ }^{2}$ have been shown to reduce loss to care. An effective intervention has not as yet been widely implemented.

The global expansion in mobile phone use, with high rates of uptake in Africa, presents new opportunities to incorporate the use of cell phones in health service delivery to help engage patients in care. In 2012, the cell phone penetration rate (number of subscribers as a proportion of the population) in Kenya was $77 \%$, with continued growth in the use of short message service (SMS). Shared-phone use is common in the region and further increases the proportion of Kenyans who use a mobile phone. ${ }^{6}$ The WelTel Kenya1 study sought to capitalise on this expansion in mobile phone access to improve healthcare delivery, and through a multisite randomised controlled trial (RCT), demonstrated that the WelTel text-messaging intervention is effective in improving adherence to medication and viral load suppression in HIV-infected individuals who had initiated ART. ${ }^{7}$ Narrative reports from participants explained that communication with their providers allowed them to feel connected with the healthcare system. It is unknown, however, whether the WelTel intervention can help prevent attrition across the cascade of care. This study will examine the effectiveness, and cost-effectiveness, of the WelTel mobile health (mHealth) intervention to improve retention in care in the early stages of HIV care in Kenya.

\section{Research hypothesis}

The WelTel patient-centred SMS service is a cost-effective strategy to improve patient retention in care in the first year following HIV diagnosis.
Study objectives

Primary objective

To determine the effect of the WelTel intervention compared with standard care on 12-month retention in care in patients newly diagnosed with HIV.

\section{Key secondary objectives}

- Determine the effect of the WelTel intervention compared with standard care on retention in stage 1 HIV care (patient returns to the clinic to receive the first CD4 count results).

- Evaluate the cost-effectiveness of the WelTel intervention on patient retention in care over a 1-year period. Employ lifetime decision analytic modelling to describe the benefit on lifetime clinical and economic outcomes of additional patients retained in care.

\section{Other secondary objectives}

- Determine the effect of the intervention on 6-month retention in care, level of engagement in care, timely initiation of ART, social support and satisfaction with care.

- Determine whether the effect of the WelTel intervention differs among important subgroups (sex, age, phone ownership, distance from clinic and baseline ART-eligibility status).

\section{METHODS AND ANALYSIS \\ Trial design}

WelTel Retain is a single site, two-arm, open, randomised, parallel-group study with a 1:1 allocation ratio.

\section{Study setting}

The trial will take place at the Kibera Community Health Centre (KCHC), an African Medical and Research Foundation (AMREF) clinic in Nairobi, Kenya, which serves over $4000 \mathrm{HIV}$-infected individuals. The health centre is a comprehensive care clinic, and there are no direct patient costs for HIV/AIDS care and treatment. The clinic is located in the heart of Kibera, one of the largest informal settlements in Africa, with an ethnically diverse population of approximately $170000{ }^{8}$ The population the clinic serves lacks or has minimal access to services such as education, water, sanitation or other public services. Transport infrastructure is minimal. In 2008, HIV prevalence among adults tested for the first time was estimated at $13 \% .^{9}$

\section{Study population}

The study population consists of adults aged 18 or over who test positive for HIV at KCHC and have not been previously assessed for ART eligibility. Individuals who test positive for HIV based on two rapid HIV tests will be referred to a research nurse. The research nurse will complete an eligibility checklist: individuals must fulfil all inclusion criteria and none of the exclusion criteria to participate. 
Figure 1 HIV cascade of care.

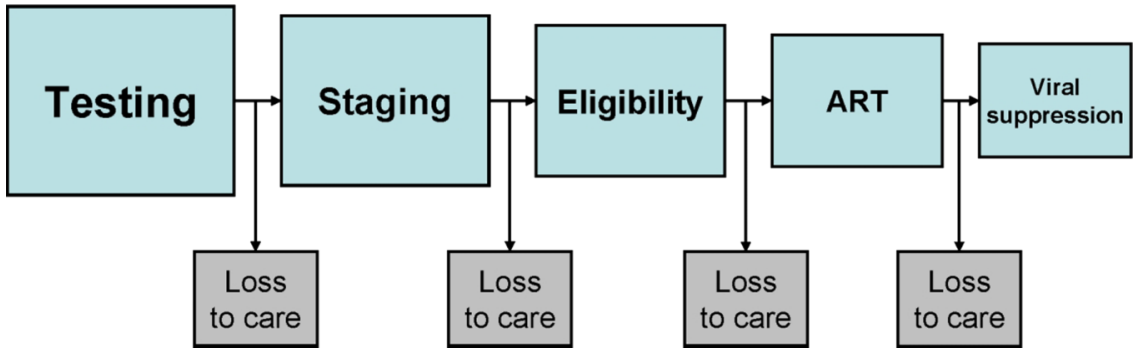

Inclusion criteria:

- Adult (18 years of age or older);

- Have an HIV test at KCHC;

- Evidence of HIV infection;

- Own or have sufficient access to a cell phone;

- Able to operate a cell phone using simple textmessaging, or have a partner, relative, etc respond on their behalf;

- Able and willing to provide informed consent to participate.

Exclusion criteria:

- Previous ART exposure;

- Currently taking ART;

- Previously assessed for ART eligibility;

- Pregnant.

Women who are pregnant at the time of diagnosis are excluded from participation because of their differing motivational factors to remain in care compared with a non-pregnant, newly diagnosed population; interventions specifically targeted to this population to enhance their retention in care and different ART treatment guidelines. Pregnant women will be enrolled in a parallel evaluation focused on PMTCT services.

\section{Interventions}

Participants will be randomly assigned to receive the WelTel text-messaging service or to usual care. The WelTel intervention involves a weekly SMS to check in on how patients are doing and provide them the opportunity to identify whether assistance is required (figure 2). For example, on Monday mornings, an automated text message from a central computer platform at the clinic will be sent to intervention arm participants asking 'Mambo?' (Kiswahili for 'How are you?'). Participants will be instructed to indicate within $48 \mathrm{~h}$ of receiving the message either that they are well (eg, 'OK' or 'Sawa') or that they have a problem (eg, 'Problem' or 'Shida'). A nurse experienced in HIV care will follow up all participants who identify a problem. Those who do not respond after $48 \mathrm{~h}$ will be called by the nurse to inquire as to their status. Participants will be informed that the SMS-support service supplements, but does not replace, existing counselling or clinical services and that all emergencies should be handled by usual means. All cell phone communication resulting from the SMS queries will be recorded by the technological platform, and the clinician will be able to enter any actions taken directly into this system.

Participants in the control and intervention groups will receive usual care at the discretion of their clinician (physician, nurse, counsellor). Standard care for HIV patients at KCHC includes psychosocial support and counselling, patient education, treatment, CD4 testing, tuberculosis and opportunistic infection screening, sexually transmitted infection screening and support, nutritional counselling, PMTCT and family planning services.

\section{Outcomes}

\section{Primary outcome}

The primary outcome is 12-month retention in care: defined as the proportion of participants retained in care at 12 months from baseline, measured by whether the participant attended a follow-up appointment in the 10-month to 14-month time frame.

\section{Key secondary outcome}

Retention in stage 1 HIV care: defined as the proportion of participants who return to the clinic to complete their first ART eligibility assessment after receiving a positive HIV test. ${ }^{10}$

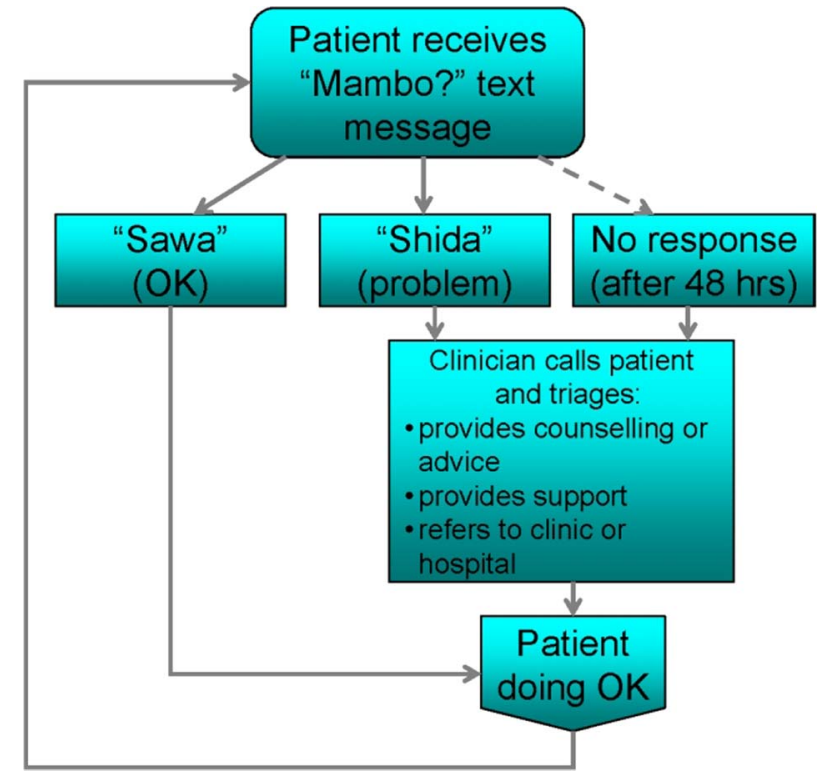

Figure 2 WelTel intervention. 


\section{Additional secondary outcomes}

- The proportion of participants who are ART eligible at baseline and who initiate ART within 3 months of HIV diagnosis.

- Time to ART initiation (for those who are ART eligible at baseline).

- Six-month retention in care (attendance at a 6-month appointment within the 5-month to 7-month time frame).

- Mean proportion of scheduled appointments kept.

- Level of engagement, defined as the proportion of participants who are non-engagers (participants who do not return after their initial visit), sporadic users (participants who attend up to $70 \%$ of their scheduled appointments) and regular users (participants who attend between $70 \%$ and $100 \%$ of their appointments).

- Level of social support (adapted Medical Outcomes Study Social Support Survey). ${ }^{11}$

- Satisfaction with care.

- Adverse events (number and grade).

- Mortality (all-cause).

A summary of primary and secondary outcomes and related hypotheses is presented in table 1 .
Rosen and Fox have proposed three stages of pre-ART HIV care, the first of which is the basis of one of our trial outcomes: retention in stage 1 HIV care. Our definition includes their proposed start point (first positive HIV test) and outcome (first ART eligibility assessment (patient returns to the clinic to receive the results of their CD4 count)). Tracking retention in stage 1 care is facilitated at the trial site because patients who test positive at the clinic commonly receive HIV care at the clinic (thereby eliminating a referral process in patients who receive a diagnosis at a stand-alone testing centre). Recent changes in clinical practice at KCHC aimed at improving patient enrolment have further simplified processes at this stage. Patients who test positive for HIV have a CD4 test at the same visit, eliminating the extra step of having to return to the clinic to have CD4 testing done. Given the truncated stage 1 care procedures at the clinic site, we have chosen to measure retention in stage 1 at care at 3 weeks, rather than at Fox $e t a l{ }^{10}$ proposed time points of 3 and 12 months.

For those initially deemed to be ART-ineligible, stage 2 HIV care is the time from completing the first ART eligibility assessment to ART eligibility, while stage 3 is the period from when a patient learns of their ART

Table 1 Outcomes, measures and methods of analysis

\begin{tabular}{|c|c|c|c|}
\hline Outcome/variable & Hypothesis & Outcome measure & Method of analysis \\
\hline \multicolumn{4}{|l|}{ Primary outcome } \\
\hline 12-month retention in care & Intervention>control & $\begin{array}{l}\text { Attends12-month clinic appointment } \\
\text { (10-14-month time frame) }\end{array}$ & $\chi^{2}$ test \\
\hline \multicolumn{4}{|l|}{ Key secondary outcomes } \\
\hline $\begin{array}{l}\text { Retention in stage } 1 \mathrm{HIV} \\
\text { care }\end{array}$ & Intervention>control & $\begin{array}{l}\text { Attends clinic to receive CD4 results } \\
\text { (within } 3 \text { weeks of positive HIV test) }\end{array}$ & $\chi^{2}$ test \\
\hline $\begin{array}{l}\text { Incremental } \\
\text { cost-effectiveness ratio }\end{array}$ & Intervention>control & $\begin{array}{l}\text { Cost per disability-adjusted life-year } \\
\text { averted }\end{array}$ & Decision analytic model \\
\hline \multicolumn{4}{|c|}{ Additional secondary outcomes } \\
\hline Timely initiation of ART & Intervention>control & $\begin{array}{l}\text { Starts ART within } 3 \text { months of eligibility } \\
\text { (for those eligible at baseline) }\end{array}$ & $\chi^{2}$ test \\
\hline Time to ART initiation & Intervention>control & ART initiation after eligible (at baseline) & $\begin{array}{l}\text { Kaplan-Meier survival } \\
\text { analysis }\end{array}$ \\
\hline 6-month retention in care & Intervention>control & $\begin{array}{l}\text { Attends 6-month clinic appointment } \\
\text { (5-7-month time frame) }\end{array}$ & $\chi^{2}$ test \\
\hline Level of engagement & Intervention>control & & \\
\hline $\begin{array}{l}\text { Proportion of scheduled } \\
\text { appointments kept }\end{array}$ & Intervention>control & $\begin{array}{l}\text { Mean proportion of scheduled } \\
\text { appointments attended }\end{array}$ & T-test \\
\hline Satisfaction with care & Intervention>control & 5-point Likert scale item & Kruskal-Wallis test \\
\hline Level of social support & Intervention>control & 5-point Likert scale item & Kruskal-Wallis test \\
\hline Quality of life & Intervention>control & SF-12 PCS and MCS scores & T test \\
\hline Death (all-cause) & Intervention>control & All-cause mortality (binary) & $\chi^{2}$ test \\
\hline \multicolumn{3}{|l|}{ Subgroup analyses } & Regression methods with \\
\hline Female vs male & Female >male & & appropriate interaction term \\
\hline Age & Younger>older & & \\
\hline Shared vs own phone & $\begin{array}{l}\text { Own phone>shared } \\
\text { phone }\end{array}$ & & \\
\hline Distance from clinic & $\leq 1 \mathrm{~h}>>1 \mathrm{~h}$ & & \\
\hline ART-eligible vs ineligible & $\bar{A} R T$ eligible>ineligible & & \\
\hline
\end{tabular}


eligibility to ART initiation. ${ }^{10}$ We will recruit participants at the time of their first positive HIV test, at which point the intervention is randomly allocated; therefore, we are precluded from measuring whether the intervention has an effect on retention in stages 2 or 3 of pre-ART HIV care, that is, because participants' baseline does not coincide with the start of the time period for later stages of pre-ART HIV care. For our primary outcome, 12-month retention in care, we will use a time frame of 10-14 months in order to accommodate differing visit schedules, which vary according to whether patients are taking ART, how recently they have started treatment (ART or co-trimoxazole) and how adherent they are to medication (ART or co-trimoxazole). Since our primary endpoint involves attendance at one appointment only, to better capture patient engagement in care we have included secondary outcomes to measure the effect of the intervention on engagement throughout the 12-month period (table 1).

With retention in care as the primary trial outcome, it is important to distinguish between retention in care and retention in clinic. This is particularly relevant, given the decentralisation and expansion of HIV care in the area. As patients have increasing options as to where they seek their care, a lack of retention in clinic is not necessarily equivalent to a lack of retention in care. A review by Geng et al suggests that retention in clinic is a poor proxy for retention in care, ${ }^{12}$ and studies have revealed significant differences in estimates of patients retained in care when a concerted effort has been made to trace those initially believed to be lost to care. ${ }^{13} \mathrm{We}$ have taken a patient perspective in our definition of retention in care, with retention defined as patients who remain in care at 12 months, even if they are accessing care outside KCHC. To determine whether participants have been retained in care, we will actively trace participants deemed to be 'lost to follow-up' in both the intervention and control groups after participants have reached the end of the follow-up period. A trained healthcare worker knowledgeable in the community setting will trace participants who will have given their consent during the enrolment process. The tracing aspect of the trial will enable us to ascertain the true status of participants (death, transfer of care or lost to care). Transferred patients active in care will be considered as retained.

\section{Sample size}

On the basis of a 1:1 allocation ratio, an $\alpha$ of 0.05 (twosided) and $80 \%$ power, it is estimated that we require 343 participants in each study arm. This calculation is based on finding a significant difference between the intervention and control arms in the primary outcome: the proportion of retained versus non-retained participants at 12 months. Sample size was calculated using the IcebergSim software version $\beta$ 4.0.3 (Practihc Coordinating Office, Oslo, Norway), a clinical trial simulator using a Monte Carlo model with 5000 simulations.
Our estimate assumes 12-month retention in $65 \%$ of control arm participants (K Kinagwi, personal communication, 2011) and a conservative estimate of $75 \%$ of patients retained at 12 months in the intervention group. This difference was selected as the smallest difference that would be important to detect and is assumed to be reasonable in the sense that an effect of this magnitude might be anticipated in this field of research.

\section{Recruitment}

Clinic staff will identify potential participants at the time of HIV diagnosis and inform them about the study (figure 3). If an individual expresses interest in the study, the clinic staff will introduce them to the research nurse who will explain the trial in further detail. The research nurse will use a checklist to assess eligibility; eligible individuals will be invited to participate and informed consent will be sought. The research nurse will maintain a recruitment $\log$ to document screened patients and report the number of participants recruited on a weekly basis. We expect to enrol 686 participants over a 1-year period. Participants will receive 150 Kenyan Shillings (KES) at the baseline visit (enrolment) and 150 KES for each subsequent study visit $(6$ and 12 months) to reimburse them for their time. They will not be reimbursed for costs associated with responding to the weekly messages. Participants will be informed through the consent process that they may withdraw from the study at any time for any reason without it affecting their medical care.

\section{Randomisation and allocation}

Randomisation of participants to the intervention or control arm will be at a 1:1 ratio, using a computergenerated randomisation list. To achieve balance between the treatment arms during the trial, blocked randomisation will be used with random sequences of different block sizes. The block sizes will not be disclosed, to ensure concealment. Allocations have been computer generated using the ralloc procedure $^{14}$ in Stata V.12.0 (Stata Corporation, College Station, Texas, USA). The individual responsible for sequence generation and allocation concealment will not be involved in the implementation of treatment assignments. Written allocation of assignments will be sealed in individual, sequentially numbered, non-resealable, opaque envelopes that will be distributed to the clinic in sufficient quantity to allocate to the targeted number of participants. After meeting inclusion criteria, consenting to participate and completing baseline assessments, participants will be immediately assigned to the randomised study arm by the research nurse who will open one of the sequentially numbered envelopes to determine allocation. Before opening the envelope, the participant's details will be written on the envelope. The assignment schedule will be kept off-site in a locked filing cabinet at the University of Nairobi. 
Figure 3 Flow of participants.

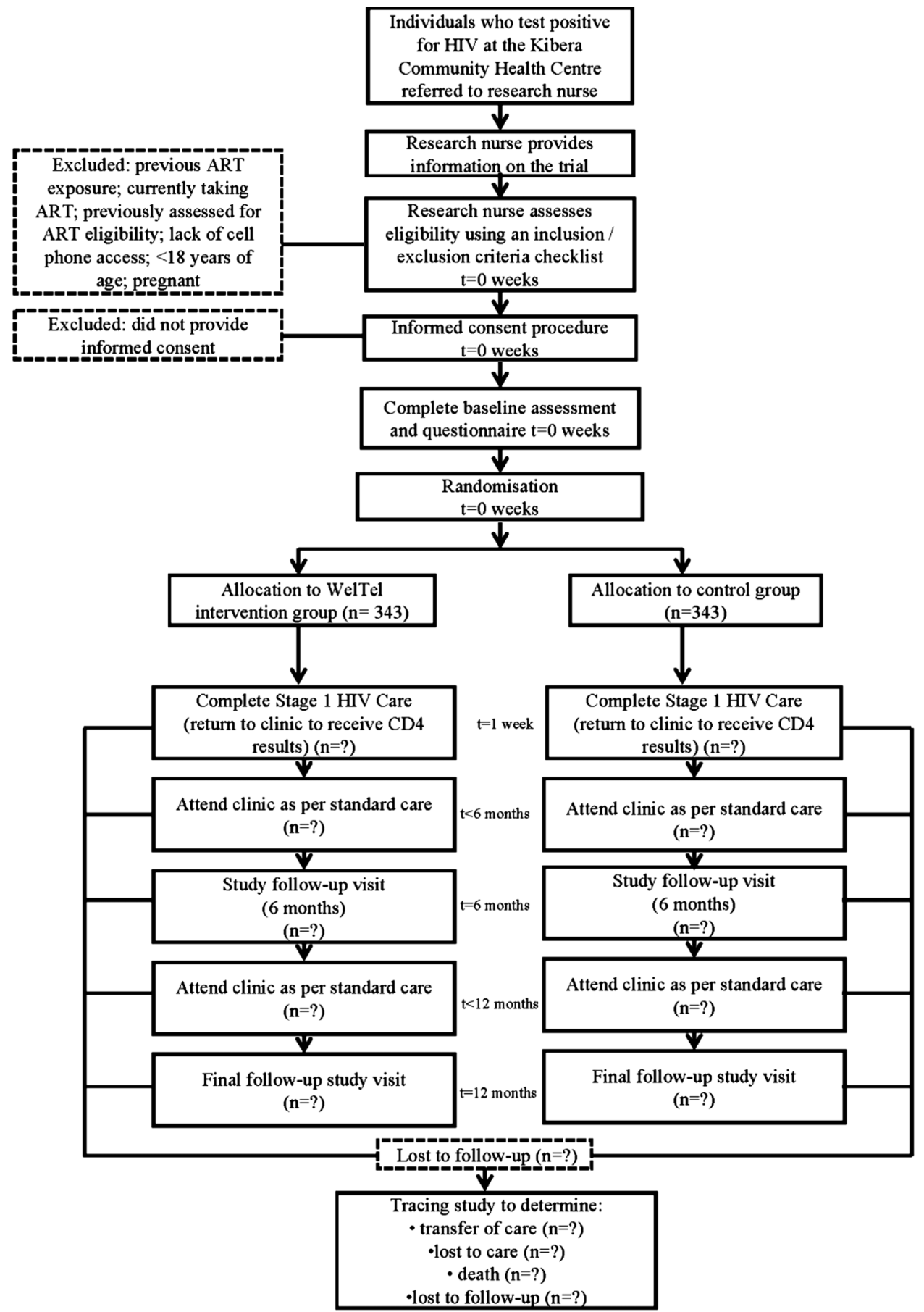

\section{Blinding}

Participating clinic staff and participants cannot be blinded because the intervention requires overt participation; however, the data analyst will be blinded.

\section{Follow-up}

Follow-up visits will occur as per routine clinical practice; each visit will be recorded. For all patients who test HIV positive, a visit is scheduled in 2 weeks to receive CD4 test results. For ART-ineligible patients, if they are adherent to co-trimoxazole and do not experience side effects, they are usually followed up once a month for the first 6 months. If patients remain adherent after
6 months, visits are scheduled once in every 2 months. ART-eligible patients are initially followed up monthly; however, the interval between visits may increase up to 3 months. In both the intervention and control groups, study procedures will occur at the 6 and 12-month clinical visits, at which time which the research nurse will administer a follow-up questionnaire. The locator form will be updated and the clinical and laboratory data retrieved. The final follow-up visit will be the clinic visit nearest to 12 months after their baseline enrolment, allowing for some natural scheduling flexibility. This visit must occur after 10 months but before 14 months from the participant's baseline visit. 
Data collection and management

WelTel's technological platform will capture the outgoing weekly SMS messages and incoming participant responses and instances of non-response. The research nurse will use the platform to record reasons why a participant responded with a problem or did not respond, and the actions that were taken. Data captured by the platform will be backed up on a daily basis.

The questionnaire and other study-related data will be paper based and entered into a Microsoft Access database at the clinic site by the data manager. Questionnaires were developed using validated measures where possible. They were translated into Kiswahili and then back-translated to English. The questionnaire is in both languages. After translation, the questionnaire was pilot tested during staff training and with patients. The data manager will check all forms for completeness. Data processing will include range and consistency checks. Any queries will be resolved promptly. Data quality will be verified by rechecking a random sample of $10 \%$ of the data collected. Participant files will be stored in a locked office at the trial site.

\section{Statistical methods}

Descriptive statistics of participants' baseline characteristics will be presented to assess their comparability. These statistics will be reported as a mean (SD) or median (first quartile, third quartile) for continuous variables, and count (per cent) for categorical variables. Baseline characteristics will include: gender, age, education, income, clinical stage, CD4 count, ART eligibility and mobile phone access.

For the primary analysis, we will compare the proportion of participants retained at 12 months in the SMS group with those in the control group using a $\chi^{2}$ test (table 1). Our analysis will be intention-to-treat; therefore, we will include all randomised patients according to the study group to which they were originally allocated, regardless of the subsequent intervention received. Results will be reported as the number of participants (with percentages) for each treatment group, the relative risk (RR) with $95 \% \mathrm{CI}$ and $\mathrm{p}$ values. We will also calculate the number needed to treat for the primary outcome. Secondary binary outcomes will be similarly analysed. For other types of secondary outcomes, we will use $t$ tests for continuous variables and Kruskal-Wallis tests for non-normally distributed variables. For time-to-event outcomes, we will analyse the data with the Kaplan-Meier approach and the Cox proportional hazards model.

Subgroup analyses will be performed by conducting the statistical analysis of the primary and secondary outcomes within the predetermined subgroups of patients. These include: sex (male vs female), age (18-29, 30-39, $40-49, \geq 50$ years of age) phone ownership (owned vs shared), distance from the clinic $(\leq 1 \mathrm{~h}$ vs $>1 \mathrm{~h})$ and ART-eligibility status at baseline (ART eligible vs ineligible $\left(\mathrm{CD} 4<350\right.$ cells $\left.\left./ \mathrm{mm}^{3}\right)\right)$. Subgroups were selected because of the potential heterogeneity in the risk of loss to care without an intervention (with men, those who are at a greater distance from the clinic, and those ineligible for ART are more likely to be lost to care) ${ }^{12}$ and potential variance in the effect of the intervention resulting from underlying differences between groups of patients in adopting a cell phone intervention (sex, age and phone ownership). We will assess whether the intervention effect is homogeneous across these subgroups by including an interaction term between the intervention allocation and subgroup-defining variables in the model. $p$ Values for the interaction tests, rather than the treatment effect within groups, will be reported. We will report all subgroup results, regardless of significance.

Missing data will be handled using multiple imputation methods. ${ }^{15}$ The criterion for statistical significance will be set at $\alpha=0.05$. The results will be reported as an estimate of the effect, corresponding to $95 \%$ CI and associated $p$ values. All $p$ values will be reported to three decimal places, with those less than 0.001 being reported as $\mathrm{p}<0.001$. The data will be analysed with up-to-date versions of Stata statistical software (Stata Corporation, College Station, Texas, USA).

\section{Data monitoring, interim analysis and stopping guidelines}

A Data and Safety Monitoring Board (DSMB), independent of the study sponsor and investigators, has been established for the trial. The board comprises a clinical trials specialist who is the Chair, a biostatistician and an expert in HIV clinical care and trials in Kenya. DSMB will periodically review safety and efficacy data. A single interim analysis will be conducted when half of the patients have reached the scheduled time of their 12-month follow-up visit. DSMB will use the Hayebittle-Peto rule, with a conservative $p$ value of $<0.001$ as a guideline to recommend that the trial be stopped. ${ }^{16} 17$ This enables us to maintain an $\alpha$ error of 0.05 for the final analysis. Recommendations for termination of the trial will be considered if there are differences in favour of one group or the other.

\section{Harms}

All adverse events occurring after enrolment until study exit (12-month follow-up for retained participants and until the end of the tracing aspect of the study for those not retained in the clinic) will be recorded. Adverse events include those directly attributable to the intervention, such as accidental disclosure of HIV status, and those resulting from participation in the trial. Potential harms will be outlined during the informed consent process and potential participants will be notified about whom these events should be reported to. An adverse event report form based on standard forms of the relevant institutional review boards (IRB) will be used as a reporting tool. The research nurse will document adverse events in a weekly study $\log$ and during follow-up visits with participants. Before the start of the trial, healthcare staff will undergo training with respect 
to the recognition and reporting of adverse events. DSMB will have access to the study data to review the occurrence of such events. Descriptive statistics will be used to report adverse events.

All unanticipated problems involving risks to human participants or others will be reported to the supporting Health \& Human Services agency head (or designee), the Office for Human Research Protections (OHRP), the research sponsor and IRBs. Unanticipated problems will be defined by OHRP criteria, as outlined in documentation on 'Guidance on Reviewing and Reporting Unanticipated Problems Involving Risks to Subjects or Others and Adverse Events'. ${ }^{18}$ Adverse events and unanticipated problems will be promptly reported to the appropriate officials in accordance with OHRP recommendations and IRB regulations. Unanticipated problems will be reported to IRBs within 2 weeks of the investigator becoming aware of the problem, unless the unanticipated problem is serious, in which case problems will be reported within 1 week. Unanticipated problems will be reported to the research sponsor, supporting agency head (or designee) and OHRP within 1 month of IRBs' receipt of the report of the problem.

\section{Qualitative aspects}

Formative and post-trial qualitative studies involving patients and healthcare workers from the study site will be conducted. The purpose of the formative phase is to gain an understanding of the potential barriers and patient knowledge of retention in care generally, and to inform any necessary adaptation of the WelTel intervention. Semistructured interviews will be conducted with five healthcare workers and 15 patients, recruited through convenience sampling. The eligibility criterion for healthcare workers is employment at KCHC. Patients will be eligible if they are a KCHC client, $\geq 18$ years, HIV positive and willing to provide consent. Interviews will be conducted in English with a translator fluent in Kiswahili and English present to assist. The interviews will be recorded, translated into English (if needed) and transcribed verbatim. Data will be analysed using an inductive thematic approach. Two researchers will read interview transcripts numerous times to identify important concepts, events and experiences. ${ }^{19}$ Once a consistent coding framework is established, transcripts will be coded using NVivo V.9 (QSR International Pty Ltd, Doncaster, Australia) software. To increase reliability, $20 \%$ of transcripts will be double coded.

The follow-up qualitative study will be conducted after the trial is complete, the purposes of which are to gain an understanding of participants' experiences during the first year of HIV care, specifically as they relate to retention and with the WelTel intervention. Theoretical sampling will be used to identify potential participant groups for recruitment. ${ }^{19}$ For example, we may recruit individuals who were initially deemed to be lost to follow-up but traced, stopped responding to the intervention, shared a phone, were treated with ART, etc, in order to gain a range of perspectives. We will recruit patients who participated in the trial and healthcare providers involved in the trial until data saturation is achieved.

\section{Cost-effectiveness evaluation}

Decision analytic modelling will be used to assess WelTel from a healthcare payer/donor perspective. An incremental analysis will evaluate the intervention compared with standard care. Direct costs of text messaging, infrastructure maintenance and technical support will be collected through trial reports and electronic reports generated by the WelTel platform. Estimates of labour costs will be derived using the activity-based costing method. ${ }^{20}$ The WHO-CHOICE database will be utilised to price labour and other resources used. ${ }^{21}$ Participant questionnaire data will include patient-specific costs and time missed from work that will be used in a secondary societal analysis.

RR of loss to care within 1 year of diagnosis will describe the benefit of WelTel compared with standard care over a short-time horizon. Modelling will extend the time horizon lifelong due to the chronic nature of HIV disease. The model will be populated using parameters identified from the literature such as rates of transmission, opportunistic infection and complications. Incremental improvements in retention will be transformed into disability-adjusted life-years (DALY) averted.

We will report the incremental cost-effectiveness ratio (ICER), in this case the ratio of the incremental cost to provide WelTel over usual care and the effect of DALY averted. The summary measure will be the incremental cost per patient retained over 1 year. ICER will also be determined for cost per DALY averted, so that we may compare WelTel to other global-health strategies and programmes. ${ }^{21}$ In accordance with WHO guidelines, we will use a discount rate of $3 \% .^{21}$ Probabilistic sensitivity analyses will be conducted on the discount rate, retention rates, costs of WelTel, costs of resources such as ART and other pertinent variables. We will perform scenario analyses including lifetime projections assuming consistent effects of WelTel over time, diminishing effects and no effect beyond 1 year. Consistent with WHO-CHOICE standards, the threshold for cost-effectiveness is three times the per capita gross domestic product (GDP) per DALY averted. ${ }^{21}$ A very cost-effective intervention is less than one time the GDP per DALY averted. ${ }^{21}$ These thresholds will be shown on an incremental costeffectiveness plane that will be constructed from the probabilistic analysis.

\section{Ancillary studies}

Participant engagement with the WelTel intervention

To better understand participant engagement with the intervention, a detailed descriptive analysis of cell phone communication will be conducted. This will include a summary of problems identified through the 
intervention and reasons why patients did not respond to the messages. Factors associated with responding to a problem and non-response will also be examined. Several mobile health (mHealth) interventions have experienced significant declines in participant response rates over time, ${ }^{22-24}$ bringing into question the longterm sustainability of these interventions. Response rates remained stable during the 12-month study period of the WelTel Kenyal trial; ${ }^{25}$ this trial will confirm whether response rates to the WelTel service are equally durable among patients at an earlier stage of HIV care. Patient-perceived benefits of and barriers to the intervention will also be examined.

\section{Retention during the first year of HIV care and factors associated with loss-to-care}

In their systematic review, Rosen and Fox indicate that studies adequately quantifying loss-to-care are scarce. ${ }^{1}$ To this end, we will conduct a prospective cohort study alongside the RCT to evaluate participant retention in the early stages of HIV care. Participants involved in the trial will be included in the cohort. To increase the generalisability of the study findings, in addition to trial participants, patients ineligible for the trial, for example, because they do not have cell phone access, will also be eligible to participate in the cohort study. Participants will be followed up for 12-months. Objectives of the cohort study are to quantify loss-to-care in the first year after diagnosis and to determine risk factors associated with loss-to-care.

\section{ETHICS AND DISSEMINATION \\ Research ethics approval}

The original study protocol, information and consent form, and baseline questionnaire were approved by the University of British Columbia Clinical Research Ethics Board (H12-00563) and the AMREF Ethics and Scientific Review Committee (P40/12). Modifications to the original trial protocol have been submitted as amendments to IRBs, and approval has been obtained. Ethical approval will be renewed on an annual basis.

\section{Consent}

After a clinic staff member introduces the trial, a trained research nurse will provide the potential participant with further details. If the participant would like to enrol, the research nurse will discuss the information in the consent form with them in the language in which the potential participant is most comfortable, English or Kiswahili. Participants will be given the opportunity to ask questions before providing written consent. Once signed, each participant will be provided with a copy of the information and consent form. Illiterate patients who wish to participate will provide consent in the presence of a literate witness; the participant's thumb print will be used in lieu of their signature.
Confidentiality

To maintain participant confidentiality, all personally identifying information will be removed from questionnaires and study documents where possible. Participants will be identified on these forms by a unique study identification number. Study documents containing personal information, for example, locator forms, informed consent forms, etc, will be kept off-site and separate from other study data. Completed questionnaires and study documents will be stored in locked filing cabinets with limited access. All personally identifying information will be removed from interview transcriptions. The risk of breach of confidentiality resulting from the text messaging intervention will be minimised since the content of the text message will not include language related to HIV. Data stored on computer databases will be password-protected and access to files will be limited.

\section{Dissemination}

Regardless of the significance, direction or magnitude of effect, we will publish our primary findings and ancillary studies in peer-reviewed journals. We will also report study findings through conference abstracts, relevant websites, at workshops and to the participating clinic staff and patients. Once all the data have been collected and cleaned, we will aim to submit the trial results for publication within 3 months.

\section{CONCLUSION}

This trial provides the opportunity to test whether the WelTel intervention is effective in earlier stages of HIV care, even before individuals start treatment. The clinic involved in the trial has several measures in place to ameliorate loss-to-care; however, retention in care is still identified as a major issue and barrier to the maximum success of their HIV care and treatment programme. Through this trial, we will be able to determine whether the WelTel text-messaging intervention, by engaging patients with the clinic on a weekly basis, is a costeffective way to help promote retention in this critical stage of HIV care.

\section{Author affiliations}

${ }^{1}$ Department of Medicine, University of British Columbia, Vancouver, Canada

${ }^{2}$ Department of Public Health Sciences, Karolinska Institutet, Stockholm, Sweden

${ }^{3}$ African Medical and Research Foundation, Nairobi, Kenya

${ }^{4}$ Faculty of Pharmaceutical Sciences, University of British Columbia,

Vancouver, Canada

${ }^{5}$ Department of Clinical Epidemiology and Biostatistics, McMaster University, Hamilton, Canada

${ }^{6}$ Clinical Prevention Services, British Columbia Centre for Disease Control, Vancouver, Canada

${ }^{7}$ WeITel International mHealth Society, Nairobi, Kenya

${ }^{8}$ Faculty of Health Sciences, University of Ottawa, Ottawa, Canada

Acknowledgements We thank the patients who participated in pretesting the questionnaire, the staff at the African Medical and Research Foundation and the Kibera Community Health Centre; the DSMB members and Jasmina Memetovic for her assistance with figures. 
Contributors MLVDK and RTL conceived the study. RTL, MLVDK, DIO, LBK, KK, LT, CM and EM secured the funding. MLVDK, RTL, DIO, LBK, KK, EM, $\mathrm{CM}, \mathrm{AME}, \mathrm{PA}, \mathrm{AP}, \mathrm{KS}$ and LT contributed to the study design. LT provided statistical expertise. MLVDK and SK designed the data collection tools. MLVDK drafted and revised the manuscript; KS and AP drafted and revised the qualitative and cost-effectiveness sections, respectively. All authors contributed critical intellectual input and approved the final manuscript.

Funding The protocol reported in this publication was supported by the National Institute of Mental Health of the National Institutes of Health under grant number R01MH097558-01. The funding source had no role in the design of the study and will not have any role during its execution, analyses, interpretation of data, or decision to submit results. The content of this publication is solely the responsibility of the authors and does not necessarily represent the official views of the National Institutes of Health. MLVDK is supported by a Canadian Institutes of Health Research (CIHR) Doctoral Foreign Study Award (October 2012), offered in partnership with the CIHR Strategy for Patient-Oriented Research and the CIHR HIV/AIDS Research Initiative.

Competing interests RTL is the founder of WeTel, a non-profit non-governmental mHealth organisation with the goal of scaling up evidence-based mHealth solutions; he has no financial stake in or salary from the organisation. For the remaining authors no conflicts of interest were declared.

Ethics approval The African Medical and Research Foundation Ethics and Scientific Review Committee and the University of British Columbia Clinical Research Ethics Board.

Provenance and peer review Not commissioned; internally peer reviewed.

\section{REFERENCES}

1. Rosen S, Fox MP. Retention in HIV care between testing and treatment in sub-Saharan Africa: a systematic review. PLoS Med 2011;8:e1001056.

2. Kohler PK, Chung MH, McGrath CJ, et al. Implementation of free cotrimoxazole prophylaxis improves clinic retention among antiretroviral therapy-ineligible clients in Kenya. AIDS 2011;25:1657-61.

3. Ford N, Kranzer K, Hilderbrand K, et al. Early initiation of antiretroviral therapy and associated reduction in mortality, morbidity and defaulting in a nurse-managed, community cohort in Lesotho. AIDS 2010;24:2645-50.

4. Boyles TH, Wilkinson LS, Leisegang $R$, et al. Factors influencing retention in care after starting antiretroviral therapy in a rural South African programme. PLOS ONE 2011;6:e19201.

5. Communications Commission of Kenya. Quarterly sector statistics report. Nairobi: Communications Commission of Kenya, 2012.

6. Wesolowski A, Eagle N, Noor AM, et al. Heterogeneous mobile phone ownership and usage patterns in Kenya. PLoS ONE 2012;7: e35319.
7. L ester RT, Ritvo P, Mills EJ, et al. Effects of a mobile phone short message service on antiretroviral treatment adherence in Kenya (WelTel Kenya1): a randomised trial. Lancet 2010;376:1838-45.

8. Kenya National Bureau of Statistics. Kenya Population and Housing Census 2009. Nairobi: Government Printer, 2010.

9. Dalal W, Feikin DR, Amolloh M, et al. Home-based HIV testing and counseling in rural and urban Kenyan communities. J Acquir Immune Defic Syndr 2013;62:e47-54.

10. Fox M, Larson B, Rosen S. Defining retention and attrition in pre-antiretroviral HIV care: proposals based on experience in Africa. Boston, MA: Center for Global Health and Development, Boston University, 2012.

11. Sherbourne CD, Stewart AL. The MOS social support survey. Soc Sci Med 1991;32:705-14.

12. Geng $E H$, Nash D, Kambugu A, et al. Retention in care among HIV-infected patients in resource-limited settings: emerging insights and new directions. Curr HIVIAIDS Rep 2010;7:234-44.

13. Geng EH, Glidden DV, Bwana MB, et al. Retention in care and connection to care among HIV-infected patients on antiretroviral therapy in Africa: estimation via a sampling-based approach. PLOS ONE 2011;6:e21797.

14. Ryan P. Random allocation of treatment in blocks. Stata J 2008;8:146.

15. Hedeker D, Mermelstein RJ, Demirtas H. Analysis of binary outcomes with missing data: missing=smoking, last observation carried forward, and a little multiple imputation. Addiction 2007;102:1564-73.

16. Peto R, Pike MC, Armitage $\mathrm{P}$, et al. Design and analysis of randomized clinical trials requiring prolonged observation of each patient. I. Introduction and design. Br J Cancer 1976;34:585-612.

17. Haybittle JL. Repeated assessment of results in clinical trials of cancer treatment. Br J Radiol 1971;44:793-7.

18. U.S. Department of Health \& Human Services. Guidance on reviewing and reporting unanticipated problems involving risks to subjects or others and adverse events. Washington, DC: U.S. Department of Health \& Human Services, 2007.

19. Padgett DK. Qualitative and mixed methods in public health. Los Angeles: Sage Publications Incorporated, 2011.

20. Kaplan RS, Anderson SR. Time-driven activity-based costing. Harv Bus Rev 2004;82:131-8.

21. Edejer Tan-Torres T, Baltussen R, Adam T, et al. eds. Making choices in health: WHO guide to cost-effectiveness analysis. Geneva: World Health Organization, 2003.

22. Hardy H, Kumar V, Doros G, et al. Randomized controlled trial of a personalized cellular phone reminder system to enhance adherence to antiretroviral therapy. AIDS Patient Care STDs 2011;25:153-61.

23. Simoni JM, Huh D, Frick PA, et al. Peer support and pager messaging to promote antiretroviral modifying therapy in Seattle: a randomized controlled trial. J Acquir Immune Defic Syndr 2009;52:465-73.

24. Franklin VL, Greene A, Waller A, et al. Patients' engagement with "Sweet Talk"-a text messaging support system for young people with diabetes. J Med Internet Res 2008;10:e20.

25. van der Kop ML, Karanja S, Thabane L, et al. In-depth analysis of patient-clinician cell phone communication during the WelTel Kenya1 antiretroviral adherence trial. PLOS ONE 2012;7:25. 